\title{
6 Konkretisierung des vorliegenden Unterfangens
}

The single most significant problem plaguing this field, in my opinion, is aptly symbolized by the parable of the elephant [...]. Language is hugely complex, and is so central to humanity that it infiltrates all aspects of human cognition, behavior, and culture. Practitioners of many different disciplines can fairly claim insight into its workings. After twenty years of studying and discussing language evolution, I conclude that many different scholars have reached valid insights about human language, some of them quite farreaching, but that no one scholar or discipline has yet achieved an adequately comprehensive overview of this complex system. All of us are still exploring the elephant of language in the darkness, all of us with only partial understanding, and each discipline will have its place in the richer description and understanding that all are seeking.

Fitch (2010: 2)

\subsection{Zum angemessenen Umgang mit der einschlägigen Literatur}

Fitch ist hinsichtlich seiner Feststellungen zum aus seiner Sicht größten Hindernis für eine adäquate Synthese des Erkenntnisstands innerhalb des modernen Forschungsfeldes der Sprachursprungsforschung prinzipiell zuzustimmen. In der Tat besteht eine große Problematik darin, dass beitragende Forscher oftmals auf die Perspektive ihrer jeweiligen Disziplin beschränkt sind und damit die Vielfalt anderer Beiträge gemeinhin nicht angemessen berücksichtigen. Das Vorgehen, dass ein Experte primär oder allein auf Basis der seiner Expertise zugrundeliegenden Disziplin auf den Forschungsgegenstand des Ursprungs von Sprachbefähigung schließt, erscheint als höchst nachvollziehbar und naheliegend - d.h. es wird beruhend auf der mit einer ebensolchen Expertise korrelierenden Perspektive sowie einer entsprechenden Konzeptbildung, Terminologie und Sensibilität vorgegangen. Die unerwünschte Folge hiervon wurde im Kontext der vorliegenden Arbeit vormals so umschrieben, dass hierdurch blinde Flecken bzw. tote Winkel gegenüber anderen Disziplinen entstehen, welche die vom jeweiligen Forscher gemachten Feststellungen und Schlussfolgerungen womöglich mehr oder weniger weitläufig untergraben. Betroffen sind hiervon freilich insbesondere diejenigen Schlussfolgerungen, welche als Extrapolationen in Bereiche außerhalb der jeweiligen Expertise zu sehen sind. Gehen die an der Gesamtdiskussion beteiligten Forscher umfänglich auf diese Weise vor, so erscheint ein produktiver interdisziplinärer Dialog als quasi unerreichbar, da beständig fehlerhafte sowie verfälschende Urteile gezogen werden und weil 
zwischen einzelnen Forschergruppen kein angemessenes gegenseitiges Verstehen hergestellt werden kann.

Eine leistungsfähige Ergründung des Sprachursprungs erfordert stattdessen - und auch hier ist Fitch zuzustimmen - eine angemessene Synthese der vielfältigen Forschungsbereiche mit ihren jeweiligen Perspektiven und Ergebnissen sowie in diesem Sinne eine außerordentliche Offenheit seitens der involvierten Forscher zugunsten einer derartigen interdisziplinären Synthese. Nur auf diese Weise ist eine Ergründung des Sprachursprungs ohne den erwähnten blinden Flecken respektive toten Winkeln $z u$ erreichen. Dennoch kann eine derartige Aufbereitung des Forschungsfeldes nicht ausschließlich, d.h. übermäßig, integrativ ausfallen. Dies fußt auf der simplen Feststellung, dass mitunter auch sich gegenseitig ausschließende Urteile innerhalb der Literatur aufzufinden sind, sodass wiederkehrend (Teil-)Positionen bzw. Forschungsergebnisse zugunsten anderer aufgegeben werden müssen, wenn ein konsistentes oder idealerweise kohärentes Gesamtbild erreicht werden soll. Eine Annäherung an die Wirklichkeit des phylogenetischen Spracherwerbs kann damit nicht allein integrativ geschehen, sondern muss wenigstens teilweise auch über den Weg kontrastiver und kritischer metawissenschaftlicher Vergleiche und entsprechender methodischer Vorgehensweisen geschehen.

\subsection{Die Forschungsfragen, Hypothesen und Mittel der vorliegenden Arbeit}

Zu diesem Zweck ist die vorliegende Arbeit so konstruiert, dass ihre Schlussfolgerungen nicht dem im Forschungsfeld üblichen Trend folgen, selektiv belegt zu sein oder aus einer singulären Perspektive gezogen zu werden. Auch versucht sich das gegebene Vorhaben nicht nach einer strikt integrativen Vorgehensweise. Stattdessen sucht das Vorgehen dieser Arbeit, die eben beschriebene Anforderung zu erfüllen und das vorliegende interdisziplinäre Forschungsfeld produktiv zu erschließen. Das zentrale Mittel hierzu ist ein Methodenkatalog, welcher eigens für das hier gegebene Vorhaben erarbeitet wurde. Dort werden aus allgemeinen metawissenschaftlichen Prinzipien Handlungsschritte abgeleitet, welche die Literaturrezeption systematisch anleiten. Auf diesem Weg sollen kognitive Verzerrungen zugunsten der einen oder anderen (Teil-)Position respektive (Teil-)Disziplin minimiert werden - mit dem Effekt, dass stattdessen eine leistungsfähige Synthese der Ergebnisse aus Einzelwissenschaften, welche die Forschungsbemühungen zum Sprachursprung informieren, möglich wird. Dieser Methodenkatalog ist nachfolgend vorzustellen und hinsichtlich seiner Einzelschritte zu diskutieren. Zuvor sollen jedoch die vordergründigen For- 
schungsfragen und Hypothesen vorgestellt werden, denn es sind ebendiese offenen Fragen bzw. begründeten, zu überprüfenden Annahmen, welche im Verlauf dieses Projektes die Notwendigkeit eines derartigen Methodenkatalogs begründeten. In diesem Sinne standen in der Frühphase des Vorhabens die drei nachfolgenden explorativen Forschungsfragen im Vordergrund.

F1 Welche Fragestellungen, Forschungsbereiche, Methoden, Argumente und Schlussfolgerungen finden sich in der einschlägigen Literatur und entsprechenden populären Sprachursprungstheorien?

F2 Zu welchen Aspekten konnte ein Konsens erreicht werden bzw. zu welchen besteht noch Uneinigkeit? Mit welcher Stärke sind die jeweiligen (Teil-)Positionen empirisch und argumentativ belegt?

F3 Wie ist das Forschungsfeld als Ganzes zu bewerten? Worin bestehen gegebenenfalls häufig auftretende Probleme und können Handlungsempfehlungen ausgesprochen werden?

Diese ersten Forschungsfragen stellten den Versuch dar, das einschlägige Forschungsfeld konzeptuell und inhaltlich in leistungsfähiger Weise zu erschließen, anstatt eine ungesteuerte Rezeption der Literatur vorzunehmen. F1 erforderte eine umfassende Literaturrecherche und -rezeption, während welcher insgesamt 40 einschlägige Handbücher, Sammelbände, Übersichtsarbeiten und Monografien herangezogen wurden. Die Auswahl geschah sowohl nach Gesichtspunkten der offenbaren Einflussname der jeweiligen Autoren und ihrer jeweiligen Veröffentlichungen als auch mit Hinblick auf eine thematisch breite Erschließung des Forschungsgegenstands. Die Ergebnisse dieses Vorgehens wurden in Form von zusammenfassenden Mitschriften sowie weiterführend abstrahierenden tabellarischen Übersichten dokumentiert. Insbesondere das letztere Vorgehen schaffte die Grundlage für eine übersichtliche direkte Vergleichbarkeit der einschlägig aktiven Autoren. Dass jenseits dieser 40 Veröffentlichungen eine weitere aktive Recherche und Rezeption eingestellt wurde, begründet sich darin, dass sich im Kontext einer fortlaufenden Rezeption eine zunehmende Tendenz zur Redundanz gegenüber des bereits Gelesenen einstellte. Damit schien das Projekt in eine zunehmend unproduktive Richtung zu laufen, weshalb in letzter Konsequenz der noch detailliert vorzustellende methodische Fokus der vorliegenden Arbeit geschaffen wurde.

Um der Fragestellung F2 gerecht werden zu können, war eine erweiterte Recherche- und Rezeptionstätigkeit vonnöten. Zwar ist es möglich, Übereinstimmung und Uneinigkeit zwischen einschlägigen Positionen im Kontext der soeben erwähnten Literaturrezeption um F1 festzustellen, jedoch reicht diese 
Recherchetätigkeit nicht für eine angemessene Einschätzung der Stärke dieser Positionen und der zugrunde liegenden Argumente aus. Hierzu ist ein Verständnis zu den einzelwissenschaftlichen Hintergründen vonnöten - d.h. sowohl ein fachliches Verständnis als auch ein metawissenschaftliches Nachvollziehen des jeweiligen Forschungsstandes. $\mathrm{Zu}$ diesem Zweck wurden rund 800 Studien und Fachartikel der jeweiligen Einzelwissenschaften herangezogen sowie Handbücher, Lehrbücher und populärwissenschaftliche Einführungen in großem Umfang rezipiert. Letztere fundierten sowohl die einführenden Kapitel - wo sie auch genannt wurden - als auch ein Verständnis zu jenen Studien und Fachartikeln, deren Rezeption eine angemessene Bewertung einschlägiger Argumentation überhaupt erst ermöglichen. In knapp zusammenfassenden Worten: Es wurde versucht, dem interdisziplinären Forschungsgegenstand in größtmöglichem Umfang gerecht zu werden. Dennoch zeigte sich F2 als eine zeitintensive und teils höchst schwierig zu beantwortende Fragestellung, sodass mancherorts Kompromisse notwendig waren bzw. schlicht eine Auswahl stattfinden musste. Eine Fortführung des vorliegenden Programms könnte dieser Limitation entgegenwirken, übersteigt jedoch die Grenzen des gegebenen Projekts.

Zuletzt wurde eine Beantwortung von F3 ursprünglich als die natürliche Kulmination all jener Bemühungen angesehen - und auch, wenn dies wenigstens teilweise auch tatsächlich der Fall ist, so erfordert eine vollumfängliche Beantwortung dieser Frage ein Level der Abstraktion, das durch eine relativ offene Rezeption der Literatur nicht gerechtfertigt aufgespannt werden kann. Auch wenn im Sinne des Vorgestellten ein vergleichsweise konstruktives Vorgehen vorlag, so offenbarte sich im Verlauf des Projektes zunehmend, dass die Vorgehensweise desselben als zu offen und vage anzusehen ist, um ihren eigenen Ansprüchen entsprechen zu können. Darin begründet sich das nachfolgend skizzierte, erweiterte Programm.

Trotz der beschriebenen Beschränkungen der ursprünglichen Forschungsfragen F1 bis F3 sowie der jeweiligen Vorgehensweisen fundierten diese ersten Schritte eine Reihe von Ergebnissen. Insbesondere F1 stellt die Grundlage zu den Ausführungen, welche im nachfolgenden Kapitel zum „Inventar der Sprachursprungsforschung“ vorzubringen sein werden. F2 und F3 führen im Kontext ihrer jeweiligen Probleme - begründet durch die Natur dieses höchst zerklüfteten interdisziplinären Forschungsfeldes - sowie auf Basis der dabei dennoch erreichten Ergebnisse zu weiterführenden Überlegungen. In dieser Weise verschob sich die zentrale Zielsetzung der vorliegenden Arbeit im weiteren Verlauf des vorliegenden Projekts zunehmend zur bereits erwähnten methodischen Zielsetzung. Die direkt folgenden Forschungsfragen erweiterten in 
dessen Sinn das ursprüngliche Programm und stellen den nun primären Fokus des gegebenen Vorhabens.

F4 In welchem Umfang und in welcher Weise können metawissenschaftliche Methoden bzw. Perspektiven auf das Forschungsfeld des Ursprungs von Sprachfähigkeit übertragen werden?

F5 Welche Schlussfolgerungen können mittels metawissenschaftlicher Methoden zu populären Fragestellungen und Diskussionen gezogen werden und inwiefern spiegeln diese Schlussfolgerungen bestehende einschlägige Arbeiten wider?

F6 Zeigen sich diese Schlussfolgerungen als miteinander vereinbar und ergeben sie ein sich stützendes Gesamtbild? ${ }^{36}$

F7 Passen bestehende Sprachursprungstheorien ganz oder teilweise zu jenen Schlussfolgerungen?

Im eben beschriebenen Sinn suchen die Forschungsfragen F4 bis F7, den Fokus der vorliegenden Arbeit in Richtung produktiver metawissenschaftlicher Methoden zu verschieben. Unter der Voraussetzung, dass dieses Programm in letzter Konsequenz als erfolgreich angesehen werden kann, so ist hierin sicherlich ein zentraler Mehrwert der vorliegenden Arbeit zu verorten. Eine Bearbeitung von F4 erforderte dabei eine weitere Rechercherunde mit Hinblick auf metawissenschaftliche Methoden innerhalb verschiedener Disziplinen, um ebendiese in unterschiedlich großem Umfang auf das vorliegende Forschungsfeld der Sprachursprungsforschung übertragen zu können. F5 baut in direkter Weise auf den Ergebnissen der Literaturrezeption im Rahmen von F2 auf, sucht nach metawissenschaftlichen Mustern im Kontext der einzelwissenschaftlichen Studien respektive Fachartikeln und kulminiert in natürlicher Weise in der Fragestellung F6. F7 greift schließlich nochmals auf die Ergebnisse von F1 zurück und sucht $\mathrm{zu}$ entscheiden, ob gegebene Gedankenkonstrukte bereits eine ausreichende Leistungsfähigkeit aufweisen. Im Sinne der soeben erläuterten Forschungsfragen sind nachfolgend zusätzlich noch die Hypothesen der vorliegenden Arbeit vorzubringen. Diese ergeben sich im Kontext jener Forschungsfragen und stützen sich teils auf unveröffentlichte Vorarbeiten und teils auf die primäre Literaturrezeption in der Frühphase des vorliegenden Vorhabens.

36 Die Implikation dieser Forschungsfrage liegt darin, dass eine entsprechende Vereinbarkeit als bestätigend hinsichtlich des gegebenen Ansatzes zu sehen wäre. Wenn ein derartiger Zustand nicht erreicht werden kann, so ist entweder die Datenlage oder die Methodologie als noch immer nicht ausreichend leistungsfähig anzusehen. 
H1 Das Forschungsfeld zeigt sich als noch immer höchst divers und zerklüftet.

H2 Ein großer Teil bestehender (Teil-)Positionen sind lediglich selektiv belegt und entsprechen noch keinem wissenschaftlichen Konsens.

H3 Metawissenschaftliche Methoden erhöhen analog $\mathrm{zu}$ anderen Forschungsfeldern die analytische Leistungsfähigkeit gegenüber dem gegebenen Forschungsgegenstand.

H4 Eine abschließende Beantwortung ist wenigstens hinsichtlich der Mehrheit der offenen Fragestellungen dennoch (noch) nicht möglich.

H5 Das Konglomerat metawissenschaftlicher Schlussfolgerungen zum Forschungsfeld stimmt mit keiner bisherigen Sprachursprungstheorie vollständig überein und zeigt sich als nicht unbedingt zu populären Strömungen passend.

\subsection{Der dem vorliegenden Projekt zugrundeliegende Methodenkatalog}

Den größten Raum des vorliegenden Kapitels nimmt der nun nachfolgende Methodenkatalog ein. Der Katalog selbst besteht aus anleitenden Anweisungen zur Rezeption von Literatur sowie deren Inhalten und soll zunächst als unkommentierte Liste vorgelegt werden, um einen einfachen Gesamtüberblick zu ermöglichen. Im Anschluss sollen die einzelnen methodischen Anweisungen jeweils für sich diskutiert und hinsichtlich ihrer Validität verteidigt werden. Bereits im Voraus ist dabei festzustellen, dass es sich dabei weitestgehend um qualitative Lösungsstrategien handelt, denn Methoden klassischer quantitativer Metastudien wie der Metaanalyse lassen sich nicht auf das gegebene, interdisziplinäre Forschungsfeld übertragen. Stattdessen sind es allgemeine Prinzipien der Metawissenschaft sowie teils konkrete Methoden qualitativer Metastudien, aus welchen sich qualitative Vorgehensweisen zum Umgang mit der einschlägigen Literatur ableiten lassen.

Weiterhin ist anzumerken, dass die einzelnen methodischen Anweisungen für sich oftmals nicht als außerordentlich innovativ zu sehen sind, sondern durch fruchtbare Vorgehensweisen in diversen metawissenschaftlichen Bereichen inspiriert wurden. So spiegeln sich einige Arbeitsschritte in einer gewissenhaften, neutralen und hochwertigen Literaturrezeption wider, während andere innerhalb qualitativ hochwertiger systematischer Übersichtsarbeiten eine nennenswerte Rolle spielen. Die primäre Innovation des vorzustellenden Methodenkatalogs ist daher nicht über einzelne darin enthaltene Anweisungen $\mathrm{zu}$ erklären, sondern vielmehr dadurch, dass aufgrund der komprimierten, 
konstruktiven und expliziten Sammlung metawissenschaftlicher Anweisungen eine leistungsfähige Schablone zur Bewertung der einschlägigen interdisziplinären Literatur entsteht. In diesem Sinne kann der nachfolgende Methodenkatalog dann als ein fruchtbares Mittel herangezogen werden, wenn die ansonsten undurchsichtige einschlägige Literatur angemessen rezipiert und bewertet werden soll - wie im weiteren Verlauf der Arbeit zu demonstrieren sein wird.

M1 Priorisiere wissenschaftlichen Konsens vor Positionen, die eine Randerscheinung darstellen.

M2 Priorisiere im Falle eines Widerspruchs basale wissenschaftliche Erkenntnisse vor speziellen Feststellungen.

M3 Priorisiere innerhalb des Raumes einer Expertise die Expertenposition vor andersartigen Positionen.

M4 Priorisiere direkte Schlussfolgerungen vor abgeleiteten Schlussfolgerungen, insbesondere wenn letztere in Geltungsbereiche anderer (Teil-)Disziplinen reichen.

M5 Priorisiere replizierte bzw. weiterführend belegte Schlussfolgerungen vor nicht replizierten bzw. nicht weiterführend belegten.

M6 Priorisiere Ergebnisse höherwertiger Studien/Papers vor weniger wertigen (klassische Qualitätskriterien und Methodenvergleich).

M6' Wenn für eine unterscheidende Bewertung im Sinne von (M6) kein ausreichendes Fachwissen vorliegt, arbeite indirekt: Verwende Reaktionen von Peers und Rezeption durch die jeweils weiterführende Forschung als Indikatoren.

M7 Priorisiere Veröffentlichungen in höherwertigen Journals vor denjenigen in weniger wertigen.

M8 Sofern gegeben, berücksichtige die gerichtete Entwicklung von Forschungsergebnissen zu einer Fragestellung über Zeit.

M9 Berücksichtige den Status bzw. die Reife der Forschung zu einer gegebenen Fragestellung.

M10 Sofern gegeben, berücksichtige die Diversität gleichberechtigter Meinungen/Hypothesen/Theorien in einer Bewertung der Situation.

M11 Suche eine angemessene Vermittlung zwischen Konservatismus und Berücksichtigung jüngerer Erkenntnisse.

M12 Überprüfe Begründungsvorgänge explizit auf selektives Vorgehen und Übergeneralisierung.

M13 Suche bei der Analyse eine Berücksichtigung der jeweils zugrundeliegenden Forscherperspektive - also der Konzepte, Terminologie und Sensibili- 
täten der (Teil-)Disziplin, welche interdisziplinäre Missverständnisse verantworten können.

Zu M1 Priorisiere wissenschaftlichen Konsens vor Positionen, die eine Randerscheinung darstellen.: Bei diesem ersten Grundsatz handelt es sich um ein intuitiv zunächst höchst eingängiges Prinzip, denn wenn sich innerhalb einer Literaturrezeption die Situation offenbart, dass die Gemeinschaft der Experten zu einem gegebenen Forschungsgegenstand eine gemeinsame, gut fundierte Meinung teilt, so erscheint es als sinnvoll, diesem Konsens zu vertrauen. Dennoch müssen Einwände ausgeräumt bzw. zusätzliche Anmerkungen gemacht werden, um hinsichtlich der vorliegenden Handlungsempfehlung Missverständnisse ausschließen zu können.

Erstens ist ein wissenschaftlicher Konsens innerhalb einer Gemeinschaft aus Experten nicht mit einer geltenden Lehrmeinung gleichzusetzen. Ein wissenschaftlicher Konsens bezieht sich gegebenenfalls konkret auf die geteilte Meinung der jeweiligen wissenschaftlichen Speerspitze, während eine breitere Lehrmeinung sich oftmals erst mit einer gewissen Verzögerung in das erweiterte Wissenschaftsverständnis und letztendlich in eine tendenziell populäre Wissenschaftskultur fortzusetzen vermag. Auf diese Weise ist möglich, dass diese beiden Phänomene nicht miteinander übereinstimmen. Derlei geschieht mit nennenswerter Häufigkeit innerhalb der Medizin, wo nicht jede neuartige Erkenntnis der einschlägigen Forschung sogleich in das medizinische Prozedere, d.h. die gängige Lehrmeinung, eingegliedert wird - aufgrund der schlichten Gründe, dass eine höchst zügige Eingliederung gemeinhin nicht möglich ist und dass eine zu schnelle Wandlung der Praxis zu praktischen Problemen und zusätzlichen Risiken führt. Wie an späterer Stelle zu zeigen sein wird, existieren derlei Phänomene auch innerhalb der Sprachursprungsforschung. In anderen Worten: Fälle, in denen sich im interdisziplinären Forschungsfeld des Ursprungs von Sprachfähigkeit eine Lehrmeinung herausbildet und auch dann von vielen einschlägig aktiven Autoren weiterhin gehalten wird, wenn der Konsens der Expertengemeinde sich bereits von diesem Punkt fortentwickelt hat und ebendieser Meinung widerspricht, existieren vielerorts in der einschlägigen Literatur.

Zweitens ist durchaus auch eine Situation denkbar, in welcher der wissenschaftliche Konsens und die gängige Lehrmeinung übereinstimmen, aber jüngere Erkenntnisse in eine neuartige Richtung zeigen. Derlei jüngeren Feststellungen wird gemeinhin völlig rechtens Skepsis entgegengebracht, denn die wissenschaftliche Praxis bzw. Meinung kann sich aus vormals bereits explizierten Gründen nicht mit jeder neuartigen Veröffentlichung schlagartig ändern, 
sondern bedarf einem angemessenen, durchaus leicht konservativen, synthetisierenden metawissenschaftlichen Urteil. Dennoch kann sich unter gegebenen Umständen freilich auch ein legitimes Spannungsfeld zwischen einem länger etablierten Konsens und der jüngeren Forschung aufmachen. In derartigen, ausreichend begründeten Fällen kann die jüngere Entwicklung unter Bezug auf andere Prinzipien wie M6, M8 oder M9 den Vorzug erhalten. In diesem Sinne gilt für das vorliegende wie auch für alle nachfolgenden Prinzipien, dass keines derselben als absolut unverletzlich anzusehen ist. Es handelt sich dabei also vielmehr um Richtlinien, welche lediglich mit guter Begründung und mit legitimem Bezug auf andere Richtlinien verletzt werden sollten, denn um unumstößliche Regeln.

$\mathrm{Zu}$ M2 Priorisiere im Falle eines Widerspruchs basale wissenschaftliche Erkenntnisse vor speziellen Feststellungen.: Dieses Prinzip des Methodenkatalogs folgt einer ähnlichen Linie wie M1. Dies gilt dahingehend, als dass diejenigen Erkenntnisse, welche für eine jeweilige Disziplin als grundlegend deklariert werden können, in einem unverhältnismäßig starken Rahmen als gesichert zu gelten haben. Basale Forschung stellt das Fundament für weiterführende Erkenntnisse und gilt bei zunehmendem Forschungsfortschritt als sukzessive stärker gesichert. Sollte nun ein neuartig entdecktes Phänomen mit derartig grundlegendem Wissen in Konflikt geraten, so liegt die natürliche Annahme nicht darin, das basale Wissenschaftsverständnis zu hinterfragen, sondern zunächst das scheinbar widersprüchliche Phänomen genauer zu betrachten. Dem folgt freilich in analoger Weise zu M1 nicht, dass derartige basale wissenschaftlichen Erkenntnisse jedem Zweifel erhaben seien oder dass neuartige Erkenntnisse prinzipiell nicht zum Überdenken basaler Forschung führen können.

Zu M3 Priorisiere innerhalb des Raumes einer Expertise die Expertenposition vor andersartigen Positionen.: Auch hinsichtlich dieses Prinzips ist keine außerordentliche Erklärungsleistung vonnöten, um die Sinnhaftigkeit desselben zu unterstreichen. Insbesondere innerhalb eines äußerst interdisziplinären Forschungsfeldes wie dem vorliegenden existiert ein Netzwerk aus Belegen, Argumenten und Schlussfolgerungen nicht nur innerhalb fachlicher Grenzen, sondern auch zwischen und über diese Grenzen hinweg. Nur allzu leicht kommt es hierbei zu Missverständnissen und Fehleinschätzungen, welche den wissenschaftlichen Austausch sowie eine interdisziplinäre Synthese erschweren. Oftmals ziehen Autoren der einschlägigen Forschung Argumente sowie Schlüsse jenseits der Grenzen ihrer eigenen Expertise und laufen dabei Gefahr, selektiv 
oder übermäßig generalisierend vorzugehen bzw. auch schlicht falsche Feststellungen zu machen. In diesem Sinne ist bei dem Versuch, interdisziplinäre Forschung zu synthetisieren, vonnöten, Expertenmeinungen im Rahmen ihrer jeweiligen Expertise zu priorisieren. Sollte damit ein Psychologe in das Feld der Linguistik wagen oder ein Linguist in dasjenige der Biologie, so sind die jeweiligen Aussagen und Schlussfolgerungen kritisch mit denjenigen einschlägiger Experten abzugleichen.

$\mathrm{Zu}$ M4 Priorisiere direkte Schlussfolgerungen vor abgeleiteten Schlussfolgerungen, insbesondere wenn letztere in Geltungsbereiche anderer (Teil-)Disziplinen reichen.: Mit dem vorliegenden Satz liegt der Versuch vor, im Nachgang zu M3 die Priorisierung zwischen Schlussfolgerungen weiter $\mathrm{zu}$ triangulieren. Dort bestand der Kern der Lösungsstrategie im Bewerten von Konflikten von Autorenpositionen, während hier die Bewertung der inhärenten Stärke von Schlussfolgerungen im Fokus steht. Gemeinhin ist der Feststellung zuzustimmen, dass mit zunehmender Entfernung von einem etablierten Zustand über mehrere Zwischenschritte bzw. Schlüsse die Sicherheit der gemachten Aussagen zunehmend abnimmt. Derlei gilt zwar nicht in einer idealen, deduktiven Schlussfolge, jedoch sind Schlüsse innerhalb der empirischen Realität im Gegensatz zu denjenigen der deduktiven Logik anfällig gegenüber störenden Faktoren oder Variablen bzw. generell aufgrund der Opazität der materiellen Realität hinsichtlich ihrer kausalen Verhältnisse - wie an früherer Stelle auch bereits ausgeführt wurde. Damit zeigt sich eine steigende Entfernung von etablierten Umständen oder Phänomenen als epistemisch zunehmend problematisch. Dies gilt aus bereits genannten Gründen umso mehr, wenn die Grenzen der eigenen Expertise überschritten werden.

$\mathrm{Zu}$ M5 Priorisiere replizierte bzw. weiterführend belegte Schlussfolgerungen vor nicht replizierten bzw. weiterführend belegten.: Sehr gerne fokussiert sich die Aufmerksamkeit der wissenschaftlichen Berichterstattung primär auf Neuerungen am Horizont des wissenschaftlichen Fortschritts. Dies erscheint intuitiv ganz natürlich, jedoch ist eine weiterführende Festigung wissenschaftlicher Erkenntnisse als wenigstens ebenso notwendig anzusehen wie diese Erschließung von Neuerungen. Erst durch dieses Vorgehen können Phänomene angemessen belegt werden, um eine Akzeptanz derselben schlussendlich begründen zu können, und viele zunächst vielversprechende Neuerungen müssen in ebendiesem Sinn nach näherer Betrachtung sukzessive zurückgenommen werden. Mithin ist dem wissenschaftlich Neuen skeptischer gegenüberzutreten als gemeinhin geschieht und triangulierende oder vertiefende Untersuchungen soll- 
ten höher gewertet werden als üblicherweise der Fall ist. Ganz in diesem Sinn leitet die vorliegende Anweisung M5 dazu an, den Status der reflektierenden wissenschaftlichen Beschäftigung zu einer Hypothese oder Schlussfolgerung als gewichtigen Faktor in einer Bewertung des Forschungsstandes aufzunehmen.

Zu M6 Priorisiere Ergebnisse höherwertiger Studien/Papers vor weniger wertigen (klassische Qualitätskriterien und Methodenvergleich).: Ganz im Sinne der Erläuterungen zur wissenschaftlichen Methode und dem Mehrwert einer Metaebene in der Wissenschaft sind auch im gegebenen Vorhaben die Ergebnisse qualitativ höherwertiger Studien denjenigen mit geringerer Qualität vorzuziehen. Eine solche Einschätzung kann synchron begründet sein, indem manche Untersuchungen schlicht gewissenhafter sind als andere, jedoch auch einen diachronen analytischen Hintergrund haben, indem jüngere Studien aufgrund technologischer oder einschlägiger methodischer Fortschritte Überlegenheit zeigen. Die jeweils konkreten Kriterien orientieren sich dabei an den Gegebenheiten und Gepflogenheiten der jeweiligen (Teil-)Disziplin.

Zu M6' Wenn für eine unterscheidende Bewertung im Sinne von (M6) kein ausreichendes Fachwissen vorliegt, arbeite indirekt: Verwende Reaktionen von Peers und Rezeption durch die jeweils weiterführende Forschung als Indikatoren.: Freilich ist es nicht möglich, hinsichtlich jeder am Forschungsgegenstand des Sprachursprungs teilhabenden (Teil-)Disziplin und jedem darin enthaltenen Forschungsfeld im Sinne von M6 vorzugehen, da das hierfür notwendige methodische Fachwissen sich als zu umfangreich und divers zeigt, um im Kontext des vorliegenden Unterfangens angemessen erlernt zu werden. Dem ist jedoch nicht die Unmöglichkeit einer adäquaten Einschätzung von Veröffentlichungen zu folgern. Eine hilfreiche Perspektive ist in aller Regel durch die Rezeption des einschlägigen Expertenkreises gegeben, indem entweder kritische Gegenstimmen geäußert werden oder im gegensätzlichen Fall Ergebnisse produktiv in die weiterführende Forschung integriert werden. Oftmals existieren Kommentare sowohl positiver als auch negativer Natur und auch Antworten durch die Erstautoren sind nicht unüblich - auch wenn in manchen Disziplinen mehr als in anderen. Durch den Einbezug derartiger informierender Perspektiven können Limitationen zum Vorgehen nach M6 oftmals umgangen werden. M7 erweitert dieses Vermögen zusätzlich.

$\mathrm{Zu}$ M7 Priorisiere Veröffentlichungen in höherwertigen Journals vor denjenigen in weniger wertigen.: Gemeinsam mit M6' sucht dieses Prinzip, auf indirektem Weg die Robustheit berücksichtigter Veröffentlichungen abzusichern, steht jedoch 
jenseits dieser zu M6' komplementären Rolle auch für sich als ein qualitätssicherndes Prinzip. Freilich ist der eigentliche Wert einer Studie bzw. allgemeiner einem Paper in ihrer respektive seiner inhärenten Qualität verankert, jedoch besteht über die Veröffentlichungsform ein unabhängiger Indikator mit Hinblick auf die Qualität der Veröffentlichung, welcher hilfreich in einem Vorhaben ist, in dem qualitativ wenig wertige Veröffentlichungen ausgeschlossen werden sollen. Gemeint sei damit: Es existieren gute Studien, Forschungsberichte und weiteres Derartiges außerhalb der großen, renommierten Journals wie Science und Nature, jedoch finden sich qualitativ ungenügende Beiträge nur selten innerhalb dieser Fachzeitschriften. Ergebnisse aus kleineren, jüngeren, weniger renommierten und hinsichtlich des Peer-Review-Prozesses weniger strengen Journals - sofern denn Peer-Review oder vergleichbare Sicherheitsnetze überhaupt im jeweiligen Prozess implementiert sind - sollten daher kritischer überprüft werden bzw. den zentraleren Veröffentlichungen nicht vorgezogen werden, auch wenn sich dort durchaus auch hochwertige Studien finden lassen.

$\mathrm{Zu}$ M8 Sofern gegeben, berücksichtige die gerichtete Entwicklung von Forschungsergebnissen zu einer Fragestellung über Zeit.: Ganz generell ist festzustellen, dass die einschlägige Forschung zu einem gegebenen Forschungsgegenstand in diachroner Hinsicht oftmals prototypischen Mustern folgt. Erste mögliche Stadien wurden bereits in der Einleitung der vorliegenden Arbeit erwähnt: $\mathrm{Zu} \mathrm{Be-}$ ginn steht die Entdeckung bzw. die erstmalige wissenschaftliche Formulierung einer Forschungsfrage, welche gegebenenfalls einen historisch mystischen bzw. religiösen Erklärungsrahmen $\mathrm{zu}$ verdrängen hat, bevor sie mit rationalen Mitteln bearbeitet werden kann. Dabei entstehen erste spekulative Erklärungsansätze und ebenso spekulative Modelle und Schlussfolgerungen - oftmals in einem Gesamtkontext sich gegenseitig ausschließender Positionen. $\mathrm{Zu}$ dem Zweck, eine Entscheidbarkeit zwischen diesen Positionen herbeizuführen, muss im weiteren Fortgang der Forschung eine methodische Selbstfindung geschehen, um eine analytische Leistungsfähigkeit gegenüber den existierenden Positionen herauszubilden und sukzessive zu steigern. Die Folge hiervon besteht in einer Spirale zwischen der Erarbeitung von Methoden, dem Erheben von Daten, der sprunghaften Veränderung bzw. Abschaffung und Neuschaffung von Positionen, der Erarbeitung neuer bzw. Verfeinerung bestehender Methoden, dem Erheben weiterer Daten und Desgleichen weiter.

Trotz der insbesondere zu Beginn eines solchen Prozesses sprunghaften Veränderungen der Forschungslandschaft sowie festgestellter Konklusionen stellt sich in diesem Prozess oftmals zügig eine Gerichtetheit der Ergebnisse ein, welche trotz der relativen Unsicherheit der Ergebnisse $\mathrm{zu}$ einem gegebenen 
Zeitpunkt prognostisch sein können. Gemeint ist hiermit, dass im sich zunehmend entwickelnden wissenschaftlichen Streben zwar wiederholt neuere Ergebnisse die jeweils älteren korrigieren und mitunter umgestalten, aber dass derartige Revisionen nicht willkürlich springen, sondern zumeist eine Konkretisierung der Forschungslandschaft sowie eine Annäherung an die tatsächliche Wirklichkeit darstellen. Damit entsteht im Rahmen einer umfassenden metawissenschaftlichen Betrachtung des Forschungsverlaufs gegebenenfalls eine leistungsfähige Diagnose zur groben Verortung einer endgültigen Antwort zum Trotz der Feststellung, dass Einzelstudien einen derartigen Punkt noch nicht erreichen konnten.

Erst über Zeit schaffen derartige primäre Untersuchungen abschließende, potenziell noch immer grobe Verortungen adäquater Schlussfolgerungen. Spätestens mit diesem doppelten Beleg mittels metawissenschaftlicher Prognose sowie primärer Forschung können Ergebnisse als provisorisch gesichert gelten. Dabei bestehen oftmals noch große Fehlerbalken, welche im Nachgang zu reduzieren sind, um einen wissenschaftlichen Konsens zu sichern sowie eine entsprechende Lehrmeinung herauszubilden - und nicht zuletzt sollte die einschlägige Forschung immer in dem Bewusstsein handeln, dass die bisherigen Bemühungen aufgrund opaker Störvariablen oder unvollendeter Perspektiven noch nicht als völlig abschließend zu sehen sind.

Ganz prinzipiell sind Hypothesen und weitere Aussagen zu einem Forschungsgegenstand zunächst entweder übermäßig generalisiert und müssen im Verlauf sich konkretisierender Forschung sukzessive zurückgenommen bzw. verengt werden oder sie sind zu konservativ und sind schrittweise zu erweitern. In beiden Fällen zeigt sich ein analytisch wertvolles Bild erst in einer angemessen umfangreichen und gewissenhaften Betrachtung des Forschungsverlaufs. In anderen Worten: Wie schon mehrmals angemerkt erlaubt nur eine stringente metawissenschaftliche Betrachtung der Forschungslandschaft im Sinne einer Loslösung vom primären Forschungsgeschehen in synchroner wie auch diachroner Weise die Schaffung eines potenziell adäquaten Urteils.

Zu M9 Berücksichtige den Status bzw. die Reife der Forschung zu einer gegebenen Fragestellung.: Bezog sich das Prinzip M8 primär auf den zeitlichen Verlauf eines Forschungsbemühens und erlaubte prognostische Schlüsse zu ebendiesem, so umfasste die Erläuterung zu M8 weiterhin auch Aspekte, welche das vorliegende M9 begründen. ${ }^{37}$ Bei der Evaluation der Stärke gegebener Feststel-

37 In diesem Sinne hat M9 eine eher synchrone analytische Funktion, während M8 eine diachrone, prognostische Funktion innehatte. 
lungen und Schlussfolgerungen seitens der Beitragenden innerhalb eines Forschungsfeldes ist vonnöten, den gegebenen Status des Forschungsfeldes in Betracht zu ziehen. Ist ein gegebener Forschungskontext ausgereift und stellt sich bereits ein umfänglicher Konsens ein, so können einschlägige Ergebnisse als recht gesichert gelten. Befindet sich jedoch ein solcher Kontext noch in der vergleichsweise chaotischen Phase der Selbstfindung, so muss einem jeden Beitrag mit einem angemessenen Vorbehalt entgegengetreten werden. Ein wohlproportionierter Ansatz zur Aufbereitung eines gegebenen Forschungsfeldes nimmt daher hierauf Rücksicht.

$\mathrm{Zu}$ M10 Sofern gegeben, berücksichtige die Diversität gleichberechtigter Meinungen/Hypothesen/Theorien in einer Bewertung der Situation.: Auch dieses Vorgehen wurde in M8 wenigstens erwähnt. In einem Stadium der Forschung, zu der (noch) nicht adäquat zwischen Positionen diskriminiert werden kann, muss ebendieses allgemeine Urteil adäquat berücksichtigt werden. Eine übermäßige Konkurrenz zwischen alternativen Ansätzen ist dem gemeinsamen Forschungsbemühen in einem solchen Kontext abträglich. Wenn kausale Verhältnisse einer Entwicklung noch größtenteils opak sind und die Antwort zu einer gegebenen Forschungsfrage damit nicht ausreichend ergründbar ist, dann sind Hypothesen und Konklusionen mit angemessener Zurückhaltung zu behandeln - wenn schon nicht von der oftmals mit großer Sicherheit und Selbstbewusstsein handelnden einschlägigen Autorschaft, dann wenigstens innerhalb des metawissenschaftlichen Programms des vorliegenden Unterfangens.

$\mathrm{Zu}$ M11 Suche eine angemessene Vermittlung zwischen Konservatismus und Berücksichtigung jüngerer Erkenntnisse.: Zum vorliegenden Prinzip ist der Gedankengang aus der Erläuterung zu M1, dass sowohl Skepsis als auch Offenheit und Unvoreingenommenheit gegenüber neuen Erkenntnissen für ein angemessenes wissenschaftliches Vorgehen notwendig sind, nochmals aufzugreifen. Prinzipiell besteht zwischen abweichenden Positionen ein Spannungsfeld, welches es nicht durch bloße Meinungsmache, sondern durch strenges methodisches Vorgehen aufzulösen gilt. In ebendiesem Sinn ist auch vorzugehen, wenn eine bestehende Lehrmeinung oder ein bestehender Konsens durch neue Veröffentlichungen kontrastiert wird.

Zu M12 Überprüfe Begründungsvorgänge explizit auf selektives Vorgehen und Übergeneralisierung.: Wie in M3 knapp diskutiert und in M8 nochmals erwähnt ist eine übermäßige Generalisierung von bzw. aus Forschungsergebnissen ganz allgemein eine umfassend bestehende Problematik innerhalb Forschungstätig- 
keiten - und dies zeigt sich insbesondere in interdisziplinären Fragestellungen wie der gegebenen. Daneben wurde mehrmals darauf hingewiesen, dass auch ein selektives Belegen eines Arguments oder einer Position unverhältnismäßig stark Gefahr läuft, zu verfälschenden Schlussfolgerungen zu führen. Aus diesem Grund muss in einer metawissenschaftlichen Aufbereitung einschlägiger Forschung wie der vorliegenden mit besonderer Gewissenhaftigkeit hinsichtlich dieser Möglichkeiten vorgegangen werden. Zeigt sich, dass eines oder beides auf einen gegebenen Argumentationsstrang zutrifft, so ist ein nennenswerter Missstand zu attestieren, denn freilich hätte die primäre Forschung bereits hierauf Rücksicht nehmen sollen.

$\mathrm{Zu}$ M13 Suche bei der Analyse eine Berücksichtigung der jeweils zugrundeliegenden Forscherperspektive - also der Konzepte, Terminologie und Sensibilitäten der (Teil-)Disziplin, welche interdisziplinäre Missverständnisse verantworten können.: Auch dieser Aspekt wurde an vorangegangener Stelle bereits angesprochen. Verschiedene Disziplinen durchliefen historisch eine mitunter ausgedehnte und noch immer sukzessive stattfindende Phase der Selbstfindung, in welcher Konzepte, Denkweisen, Sensibilitäten und die damit verbundene Terminologie in einer Weise operationalisiert wurden, die bei der Ergründung der jeweiligen Forschungsgegenstände hilfreich waren und Forschungsbemühungen zwischen Wissenschaftlern kooperativ sowie fruchtbar $\mathrm{zu}$ machen suchten. Im interdisziplinären Kontakt schlagen jedoch die systematischen Perspektivierungen beiderseitig fehl, da kein gemeinsames Vokabular existiert und beide Parteien zueinander missverständlich bzw. unverständlich erscheinen. Eine metawissenschaftliche Aufbereitung einer solchen interdisziplinären Situation muss hierauf Rücksicht nehmen und suchen, eine Vergleichbarkeit herzustellen, wo auf den ersten Blick keine zu finden ist. In einer derartigen Situation einen fruchtbaren Dialog zu vermitteln erscheint zunächst aussichtslos, ist jedoch dann erreichbar, wenn aktiv an einem angemessenen gegenseitigen Verständnis gearbeitet wird. Systematischen Übersichtsarbeiten jeglicher Form - die vorliegende Arbeit sei hinzuzurechnen - stehen in der Pflicht, den Versuch einer derartigen Vermittlung zu wagen.

Damit seien die methodischen Grundlagen sowie das generelle Vorgehen des vorliegenden Projekts ausreichend diskutiert. Anhand der soeben vorgestellten Prinzipien wurde die einschlägige Literatur umfänglich - wenn auch keinesfalls abschließend - rezipiert und synthetisiert. Das nun nachfolgende Kapitel wird einen erläuternden, recht allgemeinen Einblick in das Forschungsfeld des Ursprungs von Sprachfähigkeit ermöglichen. Im Anschluss sollen ausgewählte 
Aspekte und Forschungsbereiche der Sprachursprungsforschung im Sinne des soeben vorgestellten Methodenkatalogs aufbereitet und vorgestellt werden. Dieses Vorgehen demonstriert die Anwendbarkeit ebendieses Methodenkatalogs und schafft eine leistungsfähige Perspektive auf offene Fragen der Sprachursprungsforschung. 